\title{
Does the timing of cabergoline administration impact rates of ovarian hyperstimulation syndrome?
}

\author{
Eryn Sara Rubenfeld, MD ${ }^{1,2}$, Michael Haim Dahan, MD ${ }^{1,2}$ \\ 'Division of Gynecologic Reproductive Endocrinology and Infertility, Department of Obstetrics and Gynecology, McGill University Health Centre, Royal \\ Victoria Hospital, ${ }^{2}$ McGill University Health Centre Reproductive Centre, Montreal, Canada
}

\section{Objective}

Does the timing of cabergoline administration impact the rate of mild/moderate ovarian hyperstimulation syndrome in women with a GnRH agonist trigger?

\section{Methods}

We conducted a retrospective cohort analysis of 285 in-vitro fertilization patients at risk of OHSS who received a GnRH agonist trigger from 2011 to 2019 at McGill University Health Centre. Group 1 (Trig, n=101) began taking cabergoline $0.5 \mathrm{mg}$ orally for 7 days at the time of $\mathrm{GnRH}$ agonist trigger, while Group 2 (Retriev, n=184) started taking cabergoline on the day of oocyte retrieval. The rates of OHSS were then compared between the groups using analysis of variance and chi-square analysis, where appropriate.

\section{Results}

The baseline demographic characteristics of the two groups were similar. Trig appeared to be at a slightly higher risk of OHSS based on a significantly higher antral follicle count $(20.2 \pm 4.2$ vs. $19.0 \pm 4.3 ; P=0.02)$, higher number of stimulated follicles $>10 \mathrm{~mm}$ at trigger $(25.7 \pm 7.0$ vs. $22.8 \pm 8.3, P=0.003)$, and higher peak serum E2 level $(17,325 \pm 2,542$ vs. $14,822 \pm 3,098 ; P=0.0001)$. The Trig group had lower rates of mild and moderate OHSS $(24 \%$ vs. $36 \% ; P=0.045)$. Neither group had any patients who developed severe OHSS. Trig had fewer patients presenting with pelvic free fluid (13\% vs. $23 \% ; P=0.03)$, lower hematocrit ( $37.8 \pm 4.8 \%$ vs. $40.5 \pm 4.2 \% ; P=0.0001)$, higher albumin concentrations $(30.4 \pm 2.7$ vs. $29.5 \pm 2.0 ; P=0.01$ ), and lower potassium concentrations ( $3.9 \pm 0.5$ vs. $4.2 \pm 0.7 ; P=0.0002$ ).

\section{Conclusion}

Cabergoline at the time of trigger as compared to the time of collection should be investigated to assess its role in reducing the rates of mild/moderate OHSS.

Keywords: Cabergoline; In-vitro fertilization; Gonadotropin-releasing hormone; Ovarian hyperstimulation syndrome

\section{Introduction}

Older literature suggests that ovarian hyperstimulation syndrome (OHSS) affects in the range of $1-14 \%$ of all in-vitro fertilization cycles, with severe OHSS complicating 0.1-0.5\% of cycles [1]. The current rates of OHSS, stratified according to severity, are unknown. Preventive strategies for OHSS have been developed over the last 20 years, including the use of dopamine agonists, gonadotropin-releasing hormone $(\mathrm{GnRH})$ antagonist IVF cycles, GnRH agonist trigger, freeze-all cycles, and metformin in subjects with polycystic ovarian syndrome (PCOS) [2]. However, despite the development of GnRH agonist triggers in $\mathrm{GnRH}$ antagonist cycles and the use of dopa-
Received: 2021.02.02. Revised: 2021.04.13. Accepted: 2021.05.25. Corresponding author: Michael Haim Dahan, MD

Department of Obstetrics and Gynecology, McGill University Health Centre Reproductive Centre, 888 Boul. de Maisonneuve E \#200, Quebec H2L 4S8, Canada

E-mail: dahanhaim@hotmail.com

https://orcid.org/0000-0002-8121-7708

Articles published in Obstet Gynecol Sci are open-access, distributed under the terms of the Creative Commons Attribution Non-Commercial License (http://creativecommons. org/licenses/by-nc/3.0/) which permits unrestricted non-commercial use, distribution, and reproduction in any medium, provided the original work is properly cited.

Copyright $\odot 2021$ Korean Society of Obstetrics and Gynecology 


\title{
Obstetrics \& Gynecology Science
}

\author{
Vol. 64, No. 4, 2021
}

mine agonists, current evidence obtained from the Society for Assisted Reproductive Technology database suggests that OHSS remains a persistent issue [3]. Severe OHSS can occur even after the administration of a GnRH agonist trigger, without the addition of human chorionic gonadotropin ( $\mathrm{hCG}$ ) [4-6].

After controlled ovarian hyperstimulation $(\mathrm{COH})$ and either an hCG or GnRH agonist trigger, vascular endothelial growth factor (VEGF) released from the granulosa cells leads to an increase in vascular permeability, allowing intravascular fluid to leak into the abdominal and thoracic compartments when OHSS occurs. This fluid shift leads to intravascular hypovolemia and, if not managed appropriately, can cause significant hemoconcentration, putting patients at risk of thrombotic events. OHSS can also cause end-organ damage resulting from decreased organ perfusion [7]. To prevent OHSS, it is first necessary to understand the pathophysiology of the syndrome. VEGF, which is expressed in ovarian granulosa cells, is elevated after $\mathrm{COH}$ [8]. VEGF increases vascular permeability through interactions with its receptor, VEGF receptor 2 (VEGFR-2) [9]. One of the ways to prevent OHSS is to block the interaction between VEGF and VEGFR-2, thereby preventing an increase in vascular permeability. Cabergoline, a dopamine receptor-2 agonist, inhibits the phosphorylation of VEGFR-2 [10].

Multiple studies have demonstrated that cabergoline administration can reduce the incidence of OHSS in high-risk patients. The first use of a dopamine agonist to treat OHSS was published in 1992, using a case series of seven oliguric patients with OHSS [11]. However, there is still no standardized protocol for cabergoline administration in patients at risk of OHSS. Baumgarten et al. [12] performed a meta-analysis in 2013 and concluded that while dopamine agonists seem to help prevent OHSS, they could not draw any conclusions regarding optimal drug, dose, or regimen. A 2014 systematic review and meta-analysis of eight randomized controlled trials (RCTs) found lower rates of moderate and severe OHSS; however, the studies varied with regard to cabergoline dose, timing of initiation, length/pattern of treatment, and whether additional measures were taken to prevent OHSS [13]. Similarly, in 2016, a Cochrane Review of 16 RCTs concluded that dopamine agonists lower the rates of moderate to severe OHSS without compromising IVF outcomes; however, further research is needed to clarify the optimal dose [14]. This makes it difficult to determine the optimal timing for ca- bergoline administration, which is most effective in preventing OHSS.

A potential problem with blocking VEGF is the unknown impact that this could have on follicular growth, final oocyte maturation, and IVF outcomes. The use of cabergoline, if given too early, could theoretically impact fertilization rates, implantation rates, and ongoing pregnancy rates [15]. From animal studies, we know that the VEGFNEGFR-2 interaction is necessary for both the development of ovarian follicles to the preovulatory stage and gonadotropin-induced angiogenesis later in folliculogenesis [16]. This raises a concern as to whether giving cabergoline to prevent OHSS could inadvertently have a negative impact on oocyte maturation, endometrial angiogenesis, implantation, and pregnancy rates [8], especially if administered before oocyte retrieval. However, hCG, which is often used to trigger ovulation in IVF cycles, is known to increase the VEGF protein level by increasing VEGF mRNA concentrations in ovarian granulosa cells [17]. If, in an attempt to avoid potential detrimental effects on the developing follicles and subsequent IVF outcomes, we delay cabergoline administration until the day of oocyte retrieval instead of initiating treatment on the day of hCG trigger (36 hours earlier), it is concerning that cabergoline may be less effective in preventing OHSS. While the effect of blocking VEGFNEGFR-2 in humans remains unclear with regard to IVF success rates, there is considerable debate over the most appropriate timing of cabergoline administration when attempting to prevent OHSS [15]. Two questions arise concerning the initiation of cabergoline either before or after oocyte collection: First, does the timing of cabergoline administration have an impact on the final oocyte maturation and subsequent IVF outcomes? Second, does the timing of cabergoline administration affect its ability to prevent OHSS?

To answer the first question, a retrospective pilot study conducted in 2007 concluded that cabergoline administration starting on the day of hCG injection did not affect the rates of implantation or ongoing or term pregnancies [18]. In that study, however, the control group was not at risk of developing OHSS. In 2013, Seow et al. [15] published the first prospective RCT including 200 women, investigating the impact of the timing of cabergoline administration on final oocyte maturation, fertilization rates, and clinical outcomes. They found that the timing of cabergoline administration had no impact on any of these markers of IVF success. The OHSS rate was a secondary outcome in this study, but cabergoline 


\section{Obstetrics \& Gynecology Science}

Eryn Sara Rubenfeld, et al. Timing of cabergoline administration

seemed to have the same effect on lowering rates of moderate and severe OHSS in this study, regardless of when it was initiated. Gaafar et al. [17] conducted a case series study involving 126 patients in 2019, wherein cabergoline was administered before the hCG trigger (when the leading follicle reached a size of $15 \mathrm{~mm}$ ). They found that early administration of cabergoline was effective and safe in preventing early OHSS, and that it did not compromise pregnancy rates, although the study had no control group [17]. The results of these studies would suggest that administering cabergoline at the time of $\mathrm{hCG}$ trigger or earlier does not adversely affect IVF outcomes in humans. Two potential explanations for this are as follows: 1) the dose of cabergoline may be too low to impact the final oocyte maturation, and 2) that the high levels of VEGF produced by multiple follicles and the long duration hCG trigger may overcome the effect of partially blocking VEGF receptor function [15]. In terms of the second question, there are no studies yet with the primary goal of evaluating the timing of cabergoline administration on OHSS rates.

\section{Material and methods}

Our study was a retrospective cohort analysis of 285 patients with exuberant stimulations who underwent treatment with IVF at McGill University Health Centre from June 1, 2011, to December 31, 2019. The inclusion criteria (which ensured that all patients were at high risk of mild or moderate OHSS) were patients with PCOS [19], using the Rotterdam criteria established by the Rotterdam consensus group in 2004 [20], having 17 or more follicles greater than $10 \mathrm{~mm}$ in diameter on the day of trigger $[21,22]$, peak estradiol (E2) levels $>3,500$ $\mathrm{pg} / \mathrm{mL}$ (the equivalent of approximately $13,000 \mathrm{pmol} / \mathrm{L}$ ) [19], use of a GnRH antagonist protocol, use of a GnRH agonist trigger, and cycles in which all embryos were frozen. Exclusion criteria included patients who had a poor response to ovarian stimulation (and therefore were not at risk of developing OHSS), low E2 levels, patients who developed fewer than 17 follicles $>10 \mathrm{~mm}$, those who had an allergy to either cabergoline or other dopamine agonists, and those who were consistently on a dopamine agonist during their stimulation for a history of hyperprolactinemia.

In our institution, GnRH antagonist cycles are primarily used as good responders. In cases where there is a risk of
OHSS, a GnRH agonist trigger is used as part of a freezeall strategy. Although these patients are at a very low risk of severe OHSS, these subjects represent a legitimate group to compare outcomes based on the timing of cabergoline initiation. The ages of the patients in the study ranged from 26 to 39 years.

\section{Stimulation protocol}

All patients in our study received a $\mathrm{GnRH}$ antagonist. $\mathrm{COH}$ was achieved using either one or a combination of recombinant follicle-stimulating hormone and highly purified human menopausal gonadotropin. The starting dose of gonadotropin was chosen based on patient age, basal follicle stimulating hormone (FSH) levels, antral follicle count, body mass index, and previous response to ovarian stimulation. Adjustments to the starting dose of gonadotropin occurred based on ovarian response to the stimulation, which was monitored by transvaginal ultrasound and serial E2 measurements. Oocyte retrieval was performed using the technique described by Rose in 2014 [23].

\section{Study groups}

In this study, patients were allocated to two study groups. The first group received cabergoline $0.5 \mathrm{mg}$ orally, once daily, for 7 days, starting on the day of trigger with a GnRH agonist trigger. The second group received cabergoline $0.5 \mathrm{mg}$ orally, once daily, for 7 days, starting on the day of oocyte collection. All patients consented to a "freeze-all" strategy, where all high-quality blastocysts were frozen using a vitrification technique, and there were no fresh embryo transfers.

After oocyte collection, all patients returned to the clinic within 4-5 days for the assessment of signs and symptoms of OHSS. Examination and investigation included transvaginal and transabdominal ultrasound that intended to detect intraperitoneal free fluid and the measurement of ovarian size, and in select cases, OHSS bloodwork. Serum laboratory evaluation included hematocrit; platelets; sodium, potassium, and albumin levels. Bloodwork was ordered if the patient reported discomfort, urinary retention, weight gain, or free fluid visible on ultrasound. The OHSS classification was based on the definitions established by Navot et al. in 1992 [24]. Under this system, mild OHSS is limited mainly to abdominal distension and discomfort, and moderate OHSS includes ultrasound evidence of mild ascites. Severe OHSS includes the features of moderate OHSS plus clinical/tense ascites, ana- 


\title{
Obstetrics \& Gynecology Science
}

\author{
Vol. 64, No. 4, 2021
}

sarca, evidence of hemoconcentration, coagulation abnormalities, renal dysfunction, oliguria, and hepatic dysfunction. Finally, critical OHSS is diagnosed when there is progression of any of the features of severe OHSS, or if there is evidence of thromboembolic phenomena, renal failure, or ARDS.

\section{Statistical analysis}

The demographic data of all patients were obtained. Statistical analysis was performed with analysis of variance or chisquared $\left(X^{2}\right)$ testing where appropriate, with a $P$-value of 0.05, as the significance cutoff using SPSS 23.0.0 (IBM Corp., Armonk, NY, USA). Continuous variables were checked for normal distribution using the Kolmogorov-Smirnov test. All continuous variables were normally distributed. Stepwise multivariate logistic regression was performed to determine the risk factors for OHSS development. Variables used in the model included female age, basal serum FSH level, antral follicle count (AFC), PCOS diagnosis, number of stimulated follicles 10-14 mm in diameter at trigger, number of follicles $>10 \mathrm{~mm}$ in diameter at trigger, peak serum E2 level, number of 2 pronuclear (PN) embryos, and timing of cabergoline initiation.

\section{Results}

All 285 patients in our study were at high risk of developing OHSS, were on a GnRH antagonist protocol, and received a $\mathrm{GnRH}$ agonist trigger as part of a freeze-all strategy. The risk of developing severe OHSS was low because of the triggering medication used. Nevertheless, they were considered an excellent group to determine the role of dopamine agonists in OHSS symptomatology as related to the timing of administration. Patients in the first group began treatment with cabergoline on the day of GnRH agonist trigger (Trig), while patients in the second group started treatment with cabergoline on the day of oocyte retrieval (Retriev).

The baseline and demographic data of the patients are described in Table 1. There was a statistically significant difference between the AFCs of Trig and Retriev $(20.2 \pm 4.2$ vs.

Table 1. Baseline data comparing the two protocols

\begin{tabular}{lccc}
\hline \multirow{2}{*}{ Characteristic } & Group 1=Trig & Group 2=Retriev \\
\cline { 2 - 3 } & $\begin{array}{c}\text { Cabergoline at time of trigger } \\
(\mathbf{n}=\mathbf{1 0 1})\end{array}$ & $\begin{array}{c}\text { Cabergoline on day of collection } \\
(\mathbf{n = 1 8 4 )}\end{array}$ & P-value \\
\hline Age of female (yr) & $35.1 \pm 1.9$ & $35.0 \pm 2.4$ & 0.72 \\
Serum basal FSH level (IU/L) & $7.4 \pm 1.9$ & $7.0 \pm 2.1$ & 0.11 \\
Antral follicle count & $20.2 \pm 4.2$ & $19.0 \pm 4.3$ & 0.02 \\
PCOS diagnosis & $42(42)$ & $83(45)$ & 0.57 \\
\hline
\end{tabular}

Values are presented as mean \pm standard deviation or number (\%).

FSH, follicle stimulating hormone; PCOS, polycystic ovarian syndrome.

Table 2. Stimulation parameters comparing cabergoline initiated at trigger or at collection

\begin{tabular}{lccc}
\hline & \begin{tabular}{c} 
Group 1=Trig \\
\cline { 2 - 3 }
\end{tabular} & $\begin{array}{c}\text { Group 2=Retriev } \\
\text { trigger }(\mathbf{n}=\mathbf{1 0 1})\end{array}$ & $\begin{array}{c}\text { Cabergoline on day of } \\
\text { collection }(\mathbf{n}=\mathbf{1 8 4})\end{array}$ \\
\hline Number of follicles 10 to $14 \mathrm{~mm}$ at triggering & $15.4 \pm 5.4$ & $15.2 \pm 4.9$ & 0.75 \\
Number of follicles at least $10 \mathrm{~mm}$ at triggering & $25.7 \pm 7.0$ & $22.8 \pm 8.3$ & 0.003 \\
Peak serum E2 levels at triggering (pmol/L) & $17,325 \pm 2,542$ & $14,822 \pm 3,098$ & 0.0001 \\
Oocytes collected & $24.5 \pm 5.2(19-40)$ & $23.3 \pm 5.2(17-39)$ & 0.06 \\
2PN embryos & $14.7 \pm 6.0$ & $14.0 \pm 5.6$ & 0.33 \\
Frozen high-quality blastocysts & $4.4 \pm 2.3$ & $4.4 \pm 2.0$ & 1.0 \\
\hline
\end{tabular}

Values are presented as mean \pm standard deviation (range).

PN, pronuclear. 


\section{Obstetrics \& Gynecology Science}

Eryn Sara Rubenfeld, et al. Timing of cabergoline administration

$19.0 \pm 4.3 ; P=0.02)$. No significant differences were found in the baseline data between the two groups.

Table 2 shows the stimulation parameters and outcomes between the groups. Trig had a significantly higher number of follicles at least $10 \mathrm{~mm}$ at the time of trigger $(25.7 \pm 7.0$ vs. $22.8 \pm 8.3, P=0.003)$ as well as higher peak serum E2 levels $(17,325 \pm 2,542$ vs. $14,822 \pm 3,098 ; P=0.0001)$. Based on these data, Trig was at slightly higher risk of OHSS than Retriev. The remaining outcomes did not differ between the study groups.

The OHSS outcomes based on the timing of cabergoline administration are shown in Table 3. There were no cases of severe OHSS in either group. Overall, Trig had lower rates of OHSS than Retriev, where the rate of mild or moderate OHSS in Trig was $24 \%$, and the rate of mild or moderate OHSS in Retriev was 36\% ( $P=0.045)$. This was further demonstrated when patients in the Trig group had less pelvic free fluid (13\% vs. $23 \%$; $P=0.03)$, lower hematocrit $(37.8 \pm 4.8 \%$ vs. $40.5 \pm 4.2 \% ; P=0.0001)$, higher albumin concentrations (30.4 $\pm 2.7 \%$ vs. $29.5 \pm 2.0 \% ; P=0.01)$, lower potassium concentrations ( $3.9 \pm 0.5$ vs. $4.2 \pm 0.7 ; P=0.0002$ ), and higher sodium concentration (133.8 \pm 2.1 vs. $133.1 \pm 2.3 ; P=0.01)$. Blood workup was ordered only for patients who had signs or symptoms concerning for OHSS, which included 78 patients in Trig, and 103 patients in Retriev. The rest of the outcomes showed no significant differences between the groups.

A multivariate stepwise logistic regression analysis was performed to determine the risk factors for mild and moderate OHSS, while controlling for the effects of confounding factors. Variables used in the model included female age, basal serum FSH level, AFC, PCOS diagnosis, number of stimulated follicles 10-14 $\mathrm{mm}$ in diameter at trigger, number of follicles $>10 \mathrm{~mm}$ in diameter at trigger, peak serum E2 level, number of 2PN embryos, and timing of cabergoline initiation. The significant risk factors for mild or moderate OHSS were number of follicles $10 \mathrm{~mm}$ to $14 \mathrm{~mm}$ in diameter at the time of GnRH agonist trigger (OR 1.6, 95\% Cl: 1.4 to $1.9, P=0.001$ ), peak serum E2 level (OR 1.2, 95\% Cl: 1.05 to $1.4, P=0.001)$, PCOS diagnosis (OR $1.5,95 \% \mathrm{Cl}: 1.1$ to $1.8, P=0.02$ ), and starting cabergoline on the day of oocyte collection as compared to at the time of trigger (OR $1.4,95 \% \mathrm{Cl}: 1.2$ to 1.6 , $P=0.01)$.

Table 3. OHSS outcomes comparing cabergoline initiated at trigger or at collection

\begin{tabular}{|c|c|c|c|}
\hline & Group 1=Trig & Group 2=Retriev & \\
\hline & $\begin{array}{c}\text { Cabergoline at time of } \\
\text { trigger }(n=101)\end{array}$ & $\begin{array}{l}\text { Cabergoline on day of } \\
\text { collection }(n=184)\end{array}$ & $P$-value \\
\hline Presence of free pelvic fluid & $13(13)$ & $43(23)$ & 0.03 \\
\hline Weight gain & 0 & $2(1)$ & NS \\
\hline Discomfort/bloating & $20(20)$ & $40(22)$ & 0.70 \\
\hline Severe OHSS & 0 & 0 & - \\
\hline Mild or moderate OHSS & $24(24)$ & $66(36)$ & 0.045 \\
\hline Laboratory testing done 4-5 days after oocyte retrieval & $\mathrm{n}=78$ & $n=03$ & \\
\hline $\begin{array}{l}\text { Hemoglobin }(\mathrm{g} / \mathrm{dL}) \\
\text { Normal range }(12-16 \mathrm{~g} / \mathrm{dL})\end{array}$ & $12.6 \pm 1.6$ & $13.5 \pm 1.4$ & 0.0001 \\
\hline $\begin{array}{l}\text { Albumin (g/L) } \\
\text { Normal range (35-52 g/L) }\end{array}$ & $30.4 \pm 2.7$ & $29.5 \pm 2.0$ & 0.0001 \\
\hline $\begin{array}{l}\mathrm{Na}(\mathrm{mmol} / \mathrm{L}) \\
\text { Normal range }(133-143 \mathrm{mmol} / \mathrm{L})\end{array}$ & $133.8 \pm 2.1$ & $133.1 \pm 2.3$ & 0.01 \\
\hline $\begin{array}{l}\mathrm{K}(\mathrm{mmol} / \mathrm{L}) \\
\text { Normal range }(3.5-5 \mathrm{mmol} / \mathrm{L})\end{array}$ & $3.9 \pm 0.5$ & $4.2 \pm 0.7$ & 0.0002 \\
\hline Platelet count $\left(10^{3} /\right.$ micro L) & $205 \pm 48$ & $213 \pm 38$ & 0.12 \\
\hline
\end{tabular}

Values are presented as mean \pm standard deviation or number (\%).

OHSS, ovarian hyperstimulation syndrome; NS, not statistically significant. 


\title{
Obstetrics \& Gynecology Science
}

\author{
Vol. 64, No. 4, 2021
}

\section{Discussion}

This study investigated whether the timing of cabergoline administration affects the rate of OHSS in high-risk patients. To the best of our knowledge, this is the first study that looked specifically at when cabergoline was initiated, with the primary outcome being OHSS rates.

In a previous study that investigated clinical outcomes according to the timing of cabergoline initiation, the primary outcome was the rate of metaphase II oocytes between the study and control groups, and the incidence of moderate/severe OHSS was a secondary outcome [15]. In that study, early administration of cabergoline did not have any impact on the final oocyte maturation or fertilization rate, suggesting that starting cabergoline at the time of triggering does not have any negative impact on IVF outcomes. They also found no difference in the incidence of OHSS between their two groups, both before and after reanalyzing their data when isolating only those patients who were at the highest risk of OHSS, although they admit that their study was underpowered to draw conclusions with certainty in this regard [15].

Why did our study show that the timing of cabergoline administration did impact the rate of mild and moderate OHSS, while the previous study showed no difference? First, the two studies used different stimulation protocols and triggers. In the 2013 study, all women received a "long protocol," which employs a GnRH agonist to prevent premature ovulation and necessitates the use of hCG to trigger the final oocyte maturation [15]. In our study, all the women were on an "antagonist protocol," allowing all patients to receive a $\mathrm{GnRH}$ agonist trigger instead of hCG, drastically reducing the likelihood of OHSS. It is possible that the different trigger medications in both studies contributed to the different rates of OHSS. Second, none of our patients had fresh embryo transfers, effectively eliminating the risk of late-onset OHSS, and we reported zero cases of severe OHSS in either group. In the study by Seow et al. [15], women had a fresh embryo transfer on day 3 following oocyte retrieval, and late-onset OHSS contributed to two out of the three cases of severe OHSS in their control group. Third, in Seow et al. [15]'s study, they only reported cases of moderate or severe OHSS, whereas in our study, the two categories we looked at were mild or moderate OHSS and severe OHSS. This may explain why we found a statistically significant difference, while the previous study did not.
In our study, 24 of 101 patients in the Trig group developed mild or moderate OHSS (24\%), compared to 66 of 184 patients in the Retriev group (36\%) with a $P$-value of 0.045 , and no patients in either group developed severe OHSS. The risk difference for developing mild to moderate OHSS depending on whether cabergoline was initiated at the time of trigger or following oocyte collection was $8 \%$ (36-24\%), with a number needed to treat $12.5 \%$ (1/0.08). In other words, 12.5 patients would need to start taking cabergoline at the time of trigger to prevent one case of mild to moderate OHSS. We would expect women who received hCG triggering to have different rates of severe OHSS, as compared to what was seen in our study. The number needed to treat should be different among women who received hCG triggering. When considering the clinical relevance of decreasing rates of mild and moderate OHSS, it should be noted that mild to moderate symptoms may increase patient discomfort and anxiety, patient phone calls, and post-retrieval clinic visits, occupying more time and effort from the entire healthcare team.

Using cabergoline in a cohort that received a GnRH agonist trigger was performed to examine the role of the timing of cabergoline administration on outcomes, which may now be investigated in hCG-triggered women in future studies. Although we found no improvement in reported discomfort or in the rates of severe OHSS with different timings of cabergoline, this may not be the case in hCG-triggered cycles. Given that the only proposed change to the protocol is the timing of cabergoline initiation, and because multiple studies have shown that earlier cabergoline administration has no negative impact on IVF outcomes $[7,14,15,17,18]$, one could argue that there is no risk, and only potential benefit, to administering cabergoline starting at the time of trigger instead of following oocyte collection to reduce the rate of OHSS in moderate and high-risk patients. As demonstrated in the literature, our Trig group did not seem to have any negative outcomes related to oocyte collection and embryo development when compared to the group that received cabergoline at the time of oocyte collection. In particular, the number of oocytes collected, the number of two PN embryos, and the number of frozen high-quality blastocysts (Gardner grade BB or better) did not differ between groups in our study.

The strengths of this study are that, to the best of our knowledge, this is the first study to examine the impact of the timing of cabergoline administration on the rates of 


\section{Obstetrics \& Gynecology Science}

Eryn Sara Rubenfeld, et al. Timing of cabergoline administration

OHSS as a primary outcome. The fact that patients in both the Trig group and the Retriev group were on a GnRH antagonist protocol received a GnRH agonist trigger for final oocyte maturation, received no luteal hCG, and had all of their blastocysts cryopreserved suggests that the timing of cabergoline reduced the rates of mild and moderate OHSS in the Trig group. This finding will be important to note in future studies looking at the timing of cabergoline as a method of preventing OHSS in patients using GnRH agonist protocols and in patients using GnRH antagonist protocols who are exposed to hCG either at the time of trigger or for luteal support.

The most apparent limitation of our study is the fact that it was a retrospective cohort analysis, with the potential for undetected confounders or bias. In addition, serum anti-Mullerian hormone was unavailable at the time of data collection, as it was not routinely drawn at our clinic until recently. From a statistical standpoint, the Trig group was at higher risk of OHSS than the Retriev group. However, the Trig group ultimately demonstrated lower rates of OHSS rates. While the purpose of this study was to evaluate whether the timing of cabergoline administration affected the rate of mild or moderate OHSS, it should be noted that the use of a GnRH agonist trigger and a freeze-all strategy made it impossible to draw conclusions regarding the impact of cabergoline timing on severe OHSS as well as on late-onset OHSS. This study should be used as evidence to evaluate the outcomes of cabergoline timing on mild/moderate OHSS rates in women who are not candidates for GnRH agonist triggering.

In conclusion, our retrospective data suggest that cabergoline should be considered at the time of triggering in women at risk of OHSS. Larger, prospective studies in groups receiving an hCG trigger are needed to support these findings, so that they can be better extrapolated to the general population of IVF patients.

\section{Conflict of interest}

No potential conflict of interest relevant to this article was reported.

\section{Ethical approval}

Institutional review board permission to perform this study was obtained (number 2019-5129, approved November 23rd, 2018). The study was performed in accordance with the principles of the Declaration of Helsinki.

\section{Patient consent}

Written informed consent and the use of images from patients are not required for the publication.

\section{Funding information}

None.

\section{Acknowledgments}

We wish to thank Daniel Dahan and Yehuda Dahan for collecting the data. These data were presented as an oral presentation at the annual American Society of Reproductive Medicine meeting in 2020.

\section{References}

1. Garcia-Velasco JA. How to avoid ovarian hyperstimulation syndrome: a new indication for dopamine agonists. Reprod Biomed Online 2009;18 Suppl 2:71-5.

2. Corbett S, Shmorgun D, Claman P, Reproductive Endocrinology Infertility Committee; Special Contributor. The prevention of ovarian hyperstimulation syndrome. J Obstet Gynaecol Can 2014;36:1024-33.

3. Schirmer DA 3rd, Kulkarni AD, Zhang Y, Kawwass JF, Boulet SL, Kissin DM. Ovarian hyperstimulation syndrome after assisted reproductive technologies: trends, predictors, and pregnancy outcomes. Fertil Steril 2020;114:567-78.

4. Fatemi HM, Popovic-Todorovic B, Humaidan P, Kol S, Banker M, Devroey $\mathrm{P}$, et al. Severe ovarian hyperstimulation syndrome after gonadotropin-releasing hormone $(\mathrm{GnRH})$ agonist trigger and "freeze-all" approach in 


\section{Obstetrics \& Gynecology Science}

Vol. 64, No. 4, 2021

GnRH antagonist protocol. Fertil Steril 2014;101:1008-11.

5. Gurbuz AS, Gode F, Ozcimen N, Isik AZ. Gonadotrophinreleasing hormone agonist trigger and freeze-all strategy does not prevent severe ovarian hyperstimulation syndrome: a report of three cases. Reprod Biomed Online 2014;29:541-4.

6. Mahajan N, Gupta S, Sharma S, Rani K, Naidu P, Arora PR. Early onset ovarian hyperstimulation syndrome despite use of segmentation approach and ovarian hyperstimulation syndrome prophylaxis. J Hum Reprod Sci 2015;8:234-8.

7. Tang $H$, Hunter $T$, Hu Y, Zhai SD, Sheng $X$, Hart RJ. Cabergoline for preventing ovarian hyperstimulation syndrome. Cochrane Database Syst Rev 2012;(2):CD008605.

8. Shaltout A, Shohyab A, Youssef MA. Can dopamine agonist at a low dose reduce ovarian hyperstimulation syndrome in women at risk undergoing ICSI treatment cycles? A randomized controlled study. Eur J Obstet Gynecol Reprod Biol 2012;165:254-8.

9. Gille H, Kowalski J, Li B, LeCouter J, Moffat B, Zioncheck $T F$, et al. Analysis of biological effects and signaling properties of Flt-1 (VEGFR-1) and KDR (VEGFR-2). A reassessment using novel receptor-specific vascular endothelial growth factor mutants. J Biol Chem 2001;276:322230.

10. Kılıç N, Özdemir Ö, Başar HC, Demircan F, Ekmez F, Yücel O. Cabergoline for preventing ovarian hyperstimulation syndrome in women at risk undergoing in vitro fertilization/intracytoplasmic sperm injection treatment cycles: a randomized controlled study. Avicenna J Med 2015;5:123-7.

11. Ferraretti AP, Gianaroli L, Diotallevi L, Festi C, Trounson A. Dopamine treatment for severe ovarian hyperstimulation syndrome. Hum Reprod 1992;7:180-3.

12. Baumgarten M, Polanski L, Campbell B, Raine-Fenning N. Do dopamine agonists prevent or reduce the severity of ovarian hyperstimulation syndrome in women undergoing assisted reproduction a systematic review and metaanalysis. Hum Fertil 2013;16:168-74.

13. Leitao VM, Moroni RM, Seko LM, Nastri CO, Martins WP. Cabergoline for the prevention of ovarian hyperstimulation syndrome: systematic review and meta-analysis of randomized controlled trials. Fertil Steril 2014;101:66475.
14. Tang H, Mourad S, Zhai SD, Hart RJ. Dopamine agonists for preventing ovarian hyperstimulation syndrome. Cochrane Database Syst Rev 2016;11:CD008605.

15. Seow KM, Lin $\mathrm{YH}$, Bai $\mathrm{CH}$, Chen HJ, Hsieh BC, Huang LW, et al. Clinical outcome according to timing of cabergoline initiation for prevention of OHSS: a randomized controlled trial. Reprod Biomed Online 2013;26:562-8.

16. Zimmermann RC, Hartman T, Kavic S, Pauli SA, Bohlen $P$, Sauer MV, et al. Vascular endothelial growth factor receptor 2-mediated angiogenesis is essential for gonadotropin-dependent follicle development. J Clin Invest 2003;112:659-69.

17. Gaafar S, El-Gezary D, El Maghraby HA. Early onset of cabergoline therapy for prophylaxis from ovarian hyperstimulation syndrome (OHSS): a potentially safer and more effective protocol. Reprod Biol 2019;19:145-8.

18. Alvarez C, Alonso-Muriel I, García G, Crespo J, Bellver J, Simón $C$, et al. Implantation is apparently unaffected by the dopamine agonist Cabergoline when administered to prevent ovarian hyperstimulation syndrome in women undergoing assisted reproduction treatment: a pilot study. Hum Reprod 2007;22:3210-4.

19. Timmons D, Montrief T, Koyfman A, Long B. Ovarian hyperstimulation syndrome: a review for emergency clinicians. Am J Emerg Med 2019;37:1577-84.

20. Rotterdam ESHRE/ASRM-Sponsored PCOS Consensus Workshop Group. Revised 2003 consensus on diagnostic criteria and long-term health risks related to polycystic ovary syndrome. Fertil Steril 2004;81:19-25.

21. Seyhan A, Ata B, Polat M, Son WY, Yarali H, Dahan MH. Severe early ovarian hyperstimulation syndrome following GnRH agonist trigger with the addition of $1500 \mathrm{IU}$ hCG. Hum Reprod 2013;28:2522-8.

22. Ata B, Seyhan A, Polat M, Son WY, Yarali H, Dahan M. Reply: GnRHa trigger and modified luteal support with one bolus of hCG should be used with caution in extreme responder patients. Hum Reprod 2013;28:2594-5.

23. Rose BI. Approaches to oocyte retrieval for advanced reproductive technology cycles planning to utilize in vitro maturation: a review of the many choices to be made. J Assist Reprod Genet 2014;31:1409-19.

24. Navot D, Bergh PA, Laufer N. Ovarian hyperstimulation syndrome in novel reproductive technologies: prevention and treatment. Fertil Steril 1992;58:249-61. 